\title{
Embryonal Rhabdomyosarcoma of the Adult's Vocal Cord: A Case Report
}

\author{
Yining Li ${ }^{1}$; Zhongying Fu ${ }^{1}$; Weilun Chen ${ }^{1}$; Lixin Yang ${ }^{1}$; Wei Zhu ${ }^{1,}$ \\ ${ }^{1}$ Department of Otolaryngology and Head and Neck Surgery, The First Hospital of Jilin University, Changchun, China \\ *Corresponding Author: Wei Zhu, Department of Otolaryngology and Head and Neck Surgery, The First Hospital of Jilin University, Changchun, China. Tel: +86-13756661259, \\ Fax:+86-043184808105, E-mail: zhuwei1357@163.com
}

Received: March 25, 2015; Revised: May 27, 2015; Accepted: June 24, 2015

\begin{abstract}
Introduction: Although embryonal rhabdomyosarcomas (ERMS) represent the most common (50\% - 60\%) clinical form of rhabdomyosarcoma, these are extremely rare in adult's vocal cords. To date, only five cases of laryngeal ERMS have been reported in adults and only one of them involved the vocal cords.

Case Presentation: Herein, we reported a case of a 22-year-old woman diagnosed with ERMS, T1NOMO stage, localized at the right vocal cord. Tumor was successfully treated with a radical excision and selective neck dissection and followed-up for 5 years with no evidence of local recurrence.

Conclusions: Immunohistochemistry combined with computerized tomography (CT) and color Doppler solography of the neck and thorax should be performed. This study suggested that surgical resection was suitable for the treatment of this disease and reported the evaluations of an extremely rare case for five years.
\end{abstract}

Keywords: Rhabdomyosarcoma, Embryonal; Adult; Immunohistochemistry, Vocal Cord

\section{Introduction}

Rhabdomyosarcoma (RMS), one of the most common soft tissue sarcomas, is a malignant tumor composed of striated muscle myoblasts at different stages of differentiation (1). There are four principal histological varieties of RMS depending on the degree of cellular differentiation and maturity including embryonal, alveolar, pleomorphic and botryoid (2). Embryonal rhabdomyosarcoma (ERMS) accounted for $50 \%-60 \%$ of RMS cases (3). It was extremely rare in adults while usually occurred in children aged 3 to 12 years (4). ERMS belongs to lymph node metastasis and more than the late with blood type transfer. In the past few years, molecular biology researches showed that chromosome 11p15.5 anomaly resulted in ERMS (5). Multiple investigations affecting chromosome have been detected in ERMS cases, including knockout or insertion of specific genes associated with cancer progression (6).

Most vocal cord cancers occurred in squamous tissue (7). Spindle cell tumors accounted for only $1 \%$ of all laryngeal neoplasms (8). We reported an unusual case of adult ERMS of the vocal cord and discussed the diagnosis and treatment.

\section{Case Presentation}

A 22-year-old woman with a one-month history of hoarseness was referred to department of otolaryngology and head and neck surgery, the first hospital of Jilin University in China. She had sustained hoarseness without history of a sore throat or symptoms suggesting laryngopharyngeal reflux since July 2008. During fibrolaryngoscopy (Kawe, Germany), a lesion as a red, smooth sublobe mass $(1.5 \times 0.7$ $\times 0.5 \mathrm{~cm}$ ) was found in her right vocal cord (Figure 1 ).

In August 2008, the lesion was excised using a prop-up laryngoscope (WelchAllyn, USA). Macroscopically, the mass was brittle and vulnerable to bleeding. Fibroepithelial papilloma was diagnosed based on instant frozen pathology. The immunohistochemistry results showed MyoD-1 positivity, 20\% - 30\% Ki-67-positive cells, a few actin and smooth muscle actin-positive cells and S-100 negativity (Table 1), which confirmed that the mass was ERMS (Figure 2). Computed tomography (CT, GE Healthcare) of the lung and color Doppler sonography (Mindray, China) of the neck and abdominal regions were performed, which showed many ellipsoidal low echo-level echogenic masses in the neck. Of these masses, a $12 \times 5 \mathrm{~mm}$ mass on the left and a $10 \times 4 \mathrm{~mm}$ mass on the right were the largest and had clear margins, which confirmed the diagnosis of ERMS of the vocal cords. No lesions were detected in the liver, gall bladder, pancreas, kidneys or lungs. Under general anesthesia, selective neck dissection and right cordectomy were performed. Pathological examination showed residual ERMS with hemorrhage, necrosis, fibroplasias and foreign-body reactive giant cells in the periphery. No tumor cells were detected along the surgical margin or in the lymph nodes of the neck. We corrected the clinical diagnosis to ERMS of the right vocal cord $\left(\mathrm{T}_{1} \mathrm{~N}_{0} \mathrm{M}_{0}\right)$.

The patient was followed-up every three months during

Copyright ( 2015, Iranian Red Crescent Medical Journal. This is an open-access article distributed under the terms of the Creative Commons Attribution-NonCommercial 4.0 International License (http://creativecommons.org/licenses/by-nc/4.0/) which permits copy and redistribute the material just in noncommercial usages, provided the original work is properly cited. 
LiYetal.

the first year after surgery and every four months during 2010. From 2011 to 2012, the patient was followed-up every six months. After 2013, the 5-year follow-up visit showed no local recurrence.

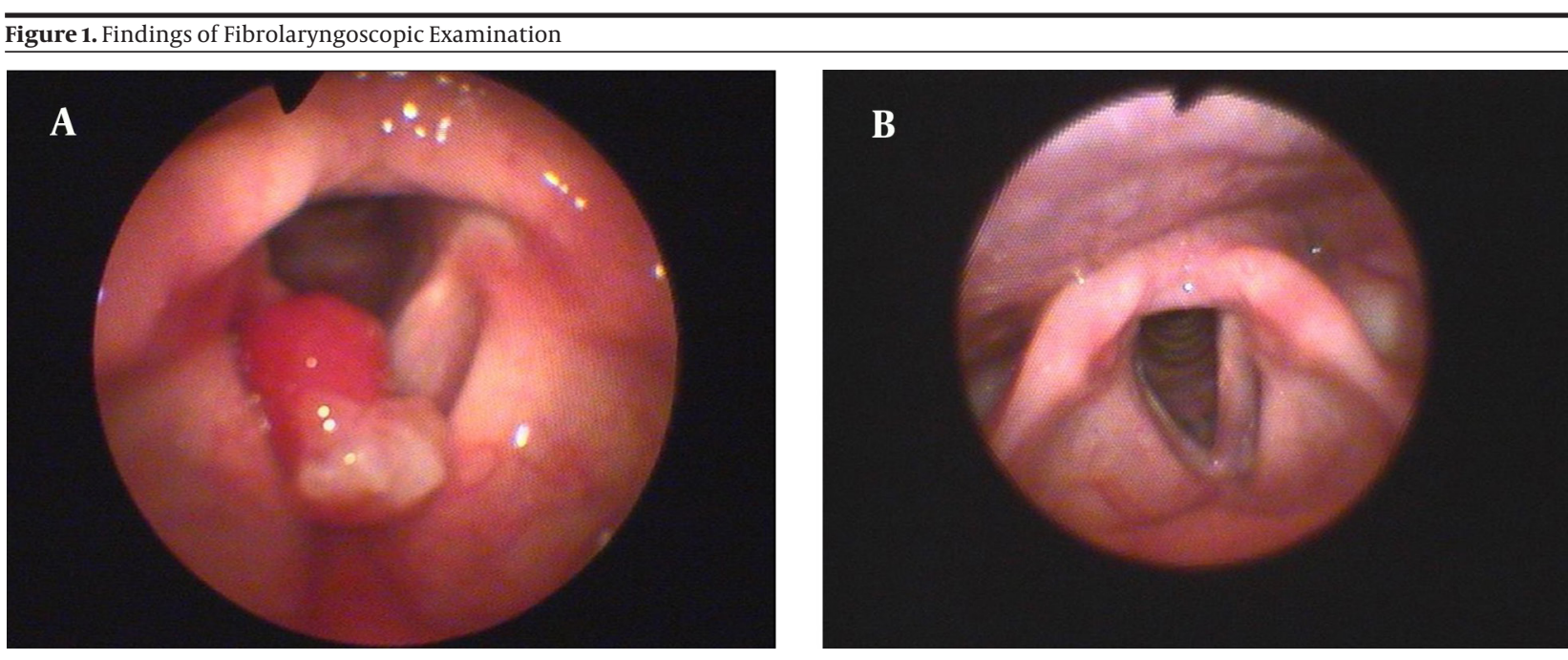

A, Fibrolaryngoscopic examination performed in August 2008 showing a $1.5 \times 0.7 \times 0.5 \mathrm{~cm}$ tumor on the right vocal cord presented as a red, smooth sublobe mass. B, Fibrolaryngoscopic examination performed in the fourth year after operation showed that the vocal cord is smooth.

\begin{tabular}{lc}
\hline Table 1. Immunochemistry Results of Each Protein & \\
\hline Proteins & Positive Rate, \% \\
\hline MyoD-1 & $70-80$ \\
Ki-67 & $20-30$ \\
Actin & $50-60$ \\
Smooth muscle actin & $50-60$ \\
S-100 & $5-10$ \\
\hline
\end{tabular}
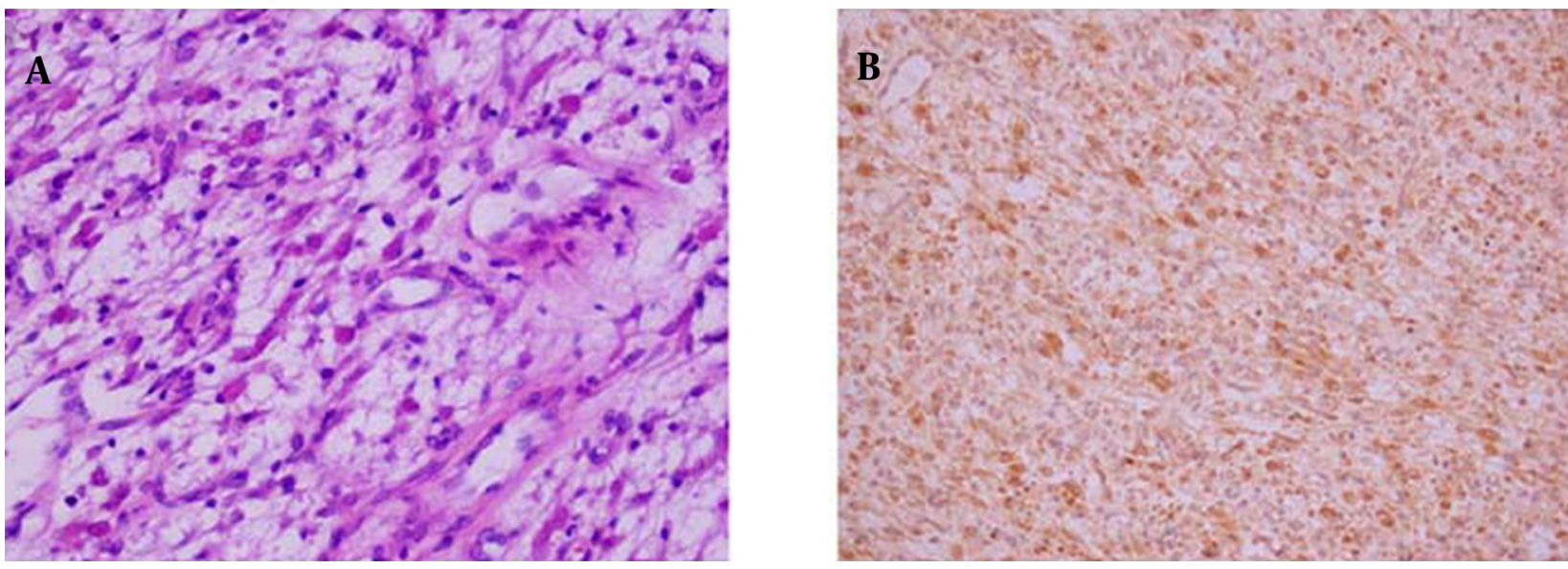

Figure 2. A, Round to Spindle-Shaped Tumor Cells With Abundant Red-Stained Cytoplasm, Loose Chromatin, Unclear Nucleoli and a Nucleus to One Side. (hematoxylin-Eosin $\times 400$ ). B, Positive MyoD1 Staining

\section{Discussion}

Rhabdomyosarcoma is the most common soft tissue sarcoma in children, which accounts for approximately 400 cases per year in the United States. Over $80 \%$ of cases in children occur before the age of 15 (9). On the other hand, adult rhabdomyosarcoma is less common and as a result, less information is available to guide clinicians to the treatment and prognosis of patient (9). In this study, we reported this interesting adult case to provide some evidence for treatment.

Many biomarkers for myogenic differentiation including myoglobin, vimentin, troponin, actin, myoglobulin etc. were developed to help diagnosis of RMS (10). How- 
ever, the low specificity and sensibility determined that detection for these biomarkers could not provide exact evidence for treatment of RMS. Yuhong at al. (11) proved that myogenic regulatory factors (MRFs) including MyoD1, myogenin, Myf5 and MRF4 had positive results in RMS, but negative in other soft tissue tumors. However, they suggested that MyoD1 could be the most efficient biomarker to determine the difference between RMS and soft tissue tumors. Our research showed that expression of actin, desmin and MyoD1 were positive based on Immunohistochemistry. Our study indicated that using an optical microscope and hematoxylin and eosin immunohistochemical staining was reliable for diagnosis of RMS.

In this case, the boundary of the tumor was limited. In addition, no metastasis was noted. Tumescent lymph nodes in the neck were detected in color Doppler ultrasonography. It was possible to diagnose the tumor in early stage, with the development of iconography, pathology and biology. We performed radical excision and selective neck dissection and no radiotherapy or chemotherapy was administered after the operation. Although the survival rate has been improved from $25 \%$ in 1970 to approximately $75 \%$ today, local tumor recurrence and metastasis remained challenging. Recent studies have shown that the recurrence rate exceeded $40 \%$ (12). In this case, the patient underwent micro-laryngoscopic evaluations, which provided no findings for malignancy and did not show metastasis. This case involved in the prognosis of ERMS of the adult vocal cord would improve dramatically with multidisciplinary treatment. However, varieties of these cases remained to be investigated.

\section{Acknowledgements}

Financial assistance was received with appreciation from Jilin International Science and Technology Cooperation Projects (Grant No. 20140414034GH).

\section{Authors' Contributions}

Study concept and design: Yining Li and Wei Zhu. Analy- sis and interpretation of data: Yining Li, Lixin Yang, Zhongying Fu, and Weilun Chen. Drafting of the manuscript: Yining Li and Wei Zhu. Critical revision of the manuscript for important intellectual content: Zhongying Fu, Wei Zhu, and Weilun Chen. Statistical analysis: Yining Li, Lixin Yang and Wei Zhu.

\section{References}

1. Poniewierska Baran A, Schneider G, Ratajczak J, Kucia M, Ratajczak MZ. Novel Evidence That Neuroblastoma and Rhabdomyosarcoma, Two Types of Small Round Blue Cell Tumors, Frequently Infiltrate Bone Marrow and Express Functional Erythropoietin Receptor (EpoR)-therapeutic Implications. Blood. 2014;124(21):4019.

2. Caporlingua F, Lapadula G, Antonelli M, Missori P. Pleomorphic rhabdomyosarcoma of the cerebellopontine angle in an adult: a review of literature. BMJ Case Rep. 2014;2014

3. Kukwa W, Wojtowicz P, Jagielska B, Sobczyk G, Kukwa A, Czarnecka AM. Laryngeal embryonal rhabdomyosarcoma in an adult - A case presentation in the eyes of geneticists and clinicians. BMC Cancer. 2011;11(1):166.

4. Wang C. Childhood rhabdomyosarcoma: recent advances and prospective views. J Dent Res. 2012;91(4):341-50.

5. Loh WJ, Scrable HJ, Livanos E, Arboleda MJ, Cavenee WK, Oshimura M, et al. Human chromosome 11 contains two different growth suppressor genes for embryonal rhabdomyosarcoma. Proc Natl Acad Sci U S A. 1992;89(5):1755-9.

6. Pazzaglia L, Chiechi A, Conti A, Gamberi G, Magagnoli G, Novello $\mathrm{C}$, et al. Genetic and molecular alterations in rhabdomyosarcoma: mRNA overexpression of MCL1 and MAP2K4 genes. Histol Histopathol. 2009;24(1):61-7.

7. Levendag PC, Teguh DN, Keskin-Cambay F, Al-Mamgani A, van Rooij P, Astreinidou E, et al. Single vocal cord irradiation: a competitive treatment strategy in early glottic cancer. Radiother Oncol. 2011;101(3):415-9.

8. Recher G. Spindle cell squamous carcinoma of the larynx. Clinico-pathological study of seven cases. J Laryngol Otol. 1985;99(9):871-9.

9. Simon JH, Paulino AC, Ritchie JM, Mayr NA, Buatti JM. Presentation, prognostic factors and patterns of failure in adult rhabdomyosarcoma. Sarcoma. 2003;7(1):1-7.

10. Sudhakar S. Alveolar Rhabdomyosarcoma on the Left Maxillary Alveolus: A Unique Presentation. J Clin Diagn Res. 2015;9(2):7-9.

11. Yuhong M, Shan W, Xiaobing D, Xiang L. The expressions and clinical significances of MyoD1 and myogenin in Rhabdomyosarcoma of childhood. J Chongqing Med Univ. 2010;12:1870-3.

12. Baker KS, Anderson JR, Link MP, Grier HE, Qualman SJ, Maurer HM, et al. Benefit of intensified therapy for patients with local or regional embryonal rhabdomyosarcoma: results from the Intergroup Rhabdomyosarcoma Study IV. J Clin Oncol. 2000;18(12):2427-34. 